\title{
Spinnaker Sail Sign Accompanied with Pneumopericardium and Pneumoperitoneum
}

\author{
Jae-kyoon Hwang ${ }^{1}$, Hyun-kyung Park ${ }^{1}$, Seok-cheol Jun ${ }^{2}$ and Hyun Ju Lee ${ }^{1^{*}}$ \\ ${ }^{1}$ Department of Pediatrics, Hanyang University College of Medicine, Seoul, Korea \\ ${ }^{2}$ Department of Radiology, Hanyang University College of Medicine, Seoul, Korea
}

"Corresponding author: Hyun Ju Lee, Department of Pediatrics, Hanyang University Seoul Hospital, 17 Haengdang-dong, Seongdong-gu, Seoul 133-792, Korea, Tel: 82-2-2290-8860; E-mail: blesslee77@hanmail.net

Received date: March 19, 2015; Accepted date: April 27, 2015; Published date: May 04, 2015

Copyright: (c) 2015 Jae-kyoon H, et al., This is an open-access article distributed under the terms of the Creative Commons Attribution License, which permits unrestricted use, distribution, and reproduction in any medium, provided the original author and source are credited.

\begin{abstract}
Air-leak syndromes include pneumomediastinum, pneumothorax, pneumopericardium, pneumoperitoneum, pulmonary interstitial emphysema and subcutaneous emphysema. Pneumothorax and pneumomediastinum occur in 1 to $2 \%$ of normal neonates and they usually has no symptoms and resolves spontaneously. Here we report sudden fatal pneumomediastinum accompanied with pneumopericardium and pneumoperitoneum in non-ventilated newborn.
\end{abstract}

Keywords: Neonate; Cardiac tamponade; Pneumomediastinum

\section{Introduction}

Spinnaker sail sign, also known as the angel wing sign, is a wedgeshaped opacity that represents the collection of air in the mediastinum. Thymic tissue is displaced from its usual location upward and laterally with gas pressure [1]. Asymptomatic pneumomediastinum has good prognosis and it resolves spontaneously. Pneumopericardium and pneumoperitoneum are rare event. But when pneumomediastinum is accompanied with pneumopericardium and symptomatic, the prognosis gets worse. Here we report sudden fatal pneumomediastinum accompanied with pneumopericardium and pneumoperitoneum in a term neonate.

\section{Case summary}

A $2900 \mathrm{~g}$ male infant was delivered to a healthy 30 -year-old woman at 38 weeks of gestation by elective repeat cesarean section. In fetal ultrasonography, there was no oligohydramnios and other anomalies. Pregnancy and delivery were uncomplicated, with Apgar scores of 9 at $1 \mathrm{~min}$, and 10 at $5 \mathrm{~min}$. Although well at birth, the infant began to present mild tachypnea at $1 \mathrm{hr}$ of age. The infant was transferred to the neonatal intermediate care unit because of mild tachypnea. At admission, chest X-ray was normal and symptoms of respiratory distress were mild and improving. Apnoea and bradycardia did not occur until 6 hrs of life, when the patient deteriorated severely with sudden onset of bradycardia, poor skin perfusion and apnoea. Arterial oxygen saturation remained more than $95 \%$ without the administration of supplemental oxygen. A chest X-ray revealed no pulmonary densities (Figure 1). Blood cultures taken at $1 \mathrm{hr}$ of age were sterile after $48 \mathrm{hrs}$ of incubation. Marked respiratory distress requiring oxygen was noted $6 \mathrm{hrs}$ after birth. The infant's clinical condition suddenly deteriorated and his heart rate remained below $100 /$ min. Endotracheal intubation was immediately performed to facilitate mechanical ventilation and to manage secondary bradycardia. Arterial oxygen saturation remained less than $70 \%$ on $100 \%$ fraction of inspired oxygen. Chest radiography obtained $6 \mathrm{hrs}$ after birth (Figure 2) showed a large quantity of air in the mediastinum causing spinnaker sail sign, extending to pneumopericardium and pneumoperitoneum. Although, a needle was immediately inserted into the second intercostal midclavicular space upon this revelation, severe bradycardia and intractable desaturation were not resolved. Chest tubes were placed in both sides for air drainage, but bradycardia and desaturation persisted without responsiveness to cardiopulmonary resuscitation. Simple needle pericardiocentesis was performed immediately to relieve cardiac tamponade via the sub-xiphoid route. Despite these interventions, intractable hypotension and bradycardia persisted. The mean arterial blood pressure decreased $1 \mathrm{hr}$ after resuscitation was ceased, the patient expired $8 \mathrm{hrs}$ after birth. The factors that led to the development of intractable pneumomediastinum remain unclear. Autopsy was not done, but there was no sign of obstructive renal anomalies.

\section{Discussion}

Pulmonary air leak in the neonate occurs when air escapes from the alveoli into extra-alveolar soft tissues or spaces [2]. The air dissects along the perivascular connective tissue, resulting in pneumothorax, pneumomediastinum, pneumopericardium, pulmonary interstitial emphysema, pneumoperitoneum, and subcutaneous emphysema [3]. Spinnaker sail sign on radiography, which occurs when thymic tissue is displaced upward and laterally by the accumulation of air in the mediastinum, cause a wedge-shaped opacity $[4,5,6]$.

The incidence of spontaneous pneumothorax and pneumomediastinum has been reported in 1 to $2 \%$ of normal term infants around the time of birth [4]. The incidence of spontaneous air leaks is not fully evaluated since the presentation can be asymptomatic, but the incidence has been shown to be increasing. 


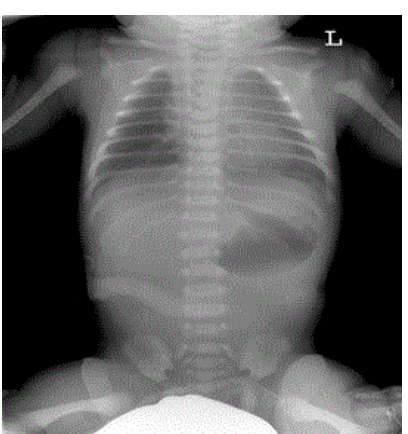

Figure 1: Chest X-ray, showing no pulmonary abnormality at $1 \mathrm{hr}$ after birth.

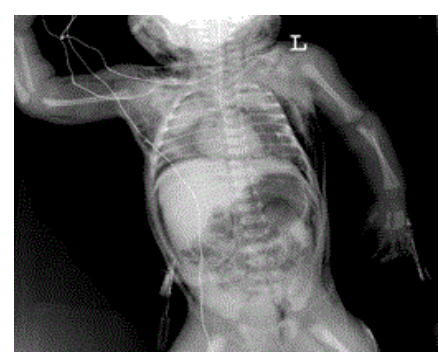

Figure 2: Supine chest radiograph showing spontaneous pneumomediastinum. Gas outlined and elevated the thymus gland, leading to "spinnaker sail sign" (arrowheads). Bilateral pneumothorax was present (short white arrow). Pneumopericardium was noted as single band of gas surrounding the left ventricle (long white arrow). Pneumoperitoneum (short black arrow) and subcutaneous emphysema (long black arrow) in the neck and chest extending from the mediastinum developed secondary to a large amount of mediastinal air.

Most cases of pneumomediastinum are asymptomatic and resolves spontaneously, although large accumulation of trapped air may cause respiratory distress such as tachypnea, grunting and cyanosis and reduce cardiac output. It is not always ominous sign. However, Spinnaker sail sign accompanied with pneumopericardium and pneumoperitoneum might be ominous sign in a term neonate. This case of a male newborn presented the sudden and dramatic deterioration of spontaneous pneumomediastinum, and that was not associated with identifiable chest trauma or positive pressure ventilation. The accumulated intrathoracic air might extend along the sheaths of pulmonary blood vessels in the hilum of the lungs and rupture into mediastinum, pericardium or extrathoracic areas. We cannot confirm that pneumomediastinum leads to pneumothorax, pneumopericardium, and pneumoperitoneum in this case. Assumption is an asymptomatic pneumomediastinum might exist before the sudden deterioration of massive pulmonary air-leak, even though no chest X-ray was followed up before the sudden episode of deterioration.

Pneumopericardium in the neonate is a rare event and occurs when air from the pleural space or mediastinum enters the pericardial sac. Pneumopericardium may present also with the abrupt onset of cardiovascular compromise, causing cardiac tamponade in the newborn infant. Infants who are asymptomatic may not need intervention for pneumopericardium [7]. However, if cardiac tamponade occurs, simple needle pericardiocentesis will be performed via the sub-xiphoid route for most cases with a life-threatening emergency. A few babies of pneumopericardium with tamponade uncontrolled by needle aspiration require pericardial tube placement for continuous drainage of the air [8]. Mortality from pneumopericardium with tamponade is high, between 70 and $90 \%$ [9]. Pneumoperitoneum can be caused by the dissection of retroperitoneal air, from pneumomediastinal into the peritoneal space. The treatment may be conservative when pneumoperitoneum will not adversely affect the patient's clinical status. Abdominal distension with upward pressure on the diaphragm may result in respiratory compromise and may reduce blood return to the heart [10]. Needle aspiration can be used as a temporizing measure or as treatment.

We report a term neonate who developed a large quantity of air in the mediastinum causing Spinnaker sail sign, accompanied with pneumopericardium and pneumoperitoneum. Highlight of this case is that spontaneous pneumomediastinum with sudden deterioration can develop in a term, unventilated infant, accompanied with tension pneumopericardium and pneumoperitoneum that may be lifethreatening in a neonate [11].

\section{Statement of financial support}

This study was supported by a Research Grant of Alumni of Pediatrics, College of Medicine, Hanyang University.

\section{References}

1. Akin K, Cizmeci MN, Kanburoglu MK, Akelma AZ, Tatli MM (2013) Angel wing sign in a neonate with pneumomediastinum. J Pediatr 163: 296.

2. Chernick V, Avery Me (1963) Spontaneous alveolar rupture at birth. Pediatrics 32: 816-824.

3. Macklin CC (1939) Transport of air along sheaths of pulmonic blood vessels from alveoli to mediastinum. Arch Intern Med 64: 913-926.

4. Davis C, Stevens G (1930) Value of routine radiographic examinations of the newborn, based on a study of 702 consecutive babies. Am J Obstet Gynecol 20: 73.

5. Correia-Pinto J, Henriques-Coelho T (2010) Images in clinical medicine. Neonatal pneumomediastinum and the spinnaker-sail sign. N Engl J Med 363: 2145 .

6. Heckmann M, Lindner W, Pohlandt F (1998) Tension pneumopericardium in a preterm infant without mechanical ventilation: a rare cause of cardiac arrest. Acta Paediatr 87: 346-348.

7. Suryawanshi P, Klimek J (2014) Preterm Neonate with Spontaneous Pneumopericardium without any Other Associated Air Leaks. J Clin Diagn Res 8: 181-182.

8. Emery RW, Lindsay WG, Nicoloff DM (1978) Placement of pericardial drainage tube for the treatment of pneumopericardium in the neonate. Ann Thorac Surg 26: 84-85.

9. Papoff P, Moretti C (2012) A Practical Approach to Neonatal Diseases, Italy.

10. Jarreau PH (2014) Pediatric and Neonatal Mechanical Ventilation: From Basics to Clinical Practice, Switzerland.

11. Renert WA, Berdon WE, Baker DH, Rose JS (1972) Obstructive urologic malformations of the fetus and infant--relation to neonatal pneumomediastinum and pneumothorax (air-block). Radiology 105: 97-105. 